\title{
Echelon Based Pose Generalization of Facial Images Approaches
}

\author{
Deepika Dubey1), G. S. Tomar2)
}

\begin{abstract}
Face recognition having many applications applied different in security, using in access control, applied on gaming, also effect by aging effect, health issues, and internet communication and so on. It has been perform on different images either they are stored in database or a new image is captured and take it as input image. Face recognition algorithms having different fields of application. The intelligent image (face) recognition system consists of various components through which human identification and recognition has been done. In real world practical applications unconstrained conditions like illumination and create variations, occlusion and expressions are to be faced by the users. in this paper discuss about the pose invariant technology also study the challenges during face recognition under unconstrained conditions like illumination and cause variations, basically it proposes several possible pose invariant technologies future directions which is excluded from the challenges used.
\end{abstract}

Keywords : face detection, recognition, image processing, PCA, KPCA, neural network.

\section{Introduction}

Face recognition system has gained popularity due to its use in security and surveillance systems. These uses of biometric information of human face are applicable easily instead of other biometric techniques like fingerprints, iris, signature recognition etc. These types of biometric techniques need physical touch and not suitable for non-collaborative people and other way, the applicability of face as biometric is comparatively easier and its working range is larger. The face is one in every of the foremost acceptable life science, and it's additionally been the foremost common methodology of recognition that human use in their visual interactions.

The problem with the authentication systems supported fingerprint, voice, and iris and

Received(January 6, 2017), Review Result(1st: January 21, 2017, 2nd: February 25, 2017), Accepted(March 10, 2017)

${ }^{1}$ MIR LAB, India email: deepika.sa1304@gmail.com

${ }^{2}$ (Corresponding Author) MIR LAB, India

email: gstomar@ieee.org 
therefore the most generated structure has been the matter of feat the info. Face recognition is field of attracting important awareness as a result of fits broad selection of potential applications [1-6]. A spread field faces recognition strategies are given during this field. It will be classified into feature-based and appearance-based techniques. Feature-based approaches withdraw options supported the belongings and geometrical associations of individual facial characteristics, as well as the eyes, eyebrows. Corner detection, iris detection, mouth, nose, and chin. However, though these approaches bank greatly on the accuracy of facial feature extraction strategies, it's been argued that existing feature-based techniques don't seem to be reliable enough for sensible applications [7][8]. Appearance-based approaches, additionally known as the holistic methodology, utilize international countenance supported high-dimensional intensity vector illustration. These strategies are wide centered on by several researchers and therefore the effectiveness of those approaches has been well tried within the literature and in business product contests like the face recognition.

The presentation of most face-recognition systems would considerably reduces if there have been many variations within the assumptions of the input image. Several algorithms are projected to unravel this downside [9]. These approaches will usually be differentiated into preprocessing, invariant feature.

Extraction and face modeling approaches. Preprocessing strategies are wide wont to extract stable options for face recognition against varied imaging conditions, reminiscent of lighting and facial expressions. Bar graph deed, Gamma correction, and exponent remodel are adopted for preprocessing in many studies [10]. Image process proficiency will think-about world process strategies.

During the face recognition or detecting a face many difficulties face by the user. First problem which is faced during face detection is frontal face [11-14]. If the frontal face is stored in image database and as input some degree is changed of the face then face is not recognized by the system this is very common problem during detecting the face. Second problem face during detection of the face is poor lightning condition, sunglasses, long/short hair, beard, low resolution etc.

Another serious problem occurred when the facial expression of the same face is to be changed in between input image and stored image. Even a smile or sadness effect the face detection system [15-17].

Face detection is used in variety of application with the help of computer technology that verify or identify human face images from the given database. Face detection algorithms basically focus on detect the exact face with the help of face detection algorithms. These 
algorithms basically focused on frontal faces. In this images are store in matric in pixel form. Image is match every pixel bit wise input image with stored database image. Any change in the bit due to problem occurred during taking input image will deny the matching process.

\section{Literature Review}

R. Brunelli and T. Poggio [1] author proposes template matching of the face and compare it with the feature of the face and the recognition of the face is possible. After getting the result compare template verses features and getting best algorithm.

Jian Kang Wu and A. D. Narasimhalu [2] explained a face recognition system throughout an investigation, officers typically need to kind through many images to spot a suspect. To help this task enforced a versatile info system that may retrieve faces exploitation personal info, fuzzy and free-text descriptors, and classification trees.

Nikolaus F. Troje and Heinrich H. Bülthoff, [3] author proposes a face recognition isn't dead invariant to create and viewpoint changes. gift work provides such information employing a high angular resolution and an oversized vary of poses it additionally emphasize the roles of the training read and therefore the testing read within the recognition experiment.

Yael Adini et al., [4] explained a face recognition system during which facing the matter in between an equivalent face of variation within the pictures. A standard technique to replace image distortion thanks to switches within the illumination status has been used for image representations that square measure comparatively incurious to that dissimilarity.

J. Ben-Arie and D. Nandy, [5] author make a case for a completely unique methodology for showing 3D objects that bring together for viewer and paradigmatic which focused object representations is bestowed. A different 3D frequency-domain illustration, known as meter frequency illustration (VFR), encloses every spatial model of the item and a time of its views within the similar organization.

Toshiaki Kondo and Hong Yan, [6] a system for automatic external body part detection and recognition is bestowed. The procedure consists of the many steps like The Haar ripple transforms, facial edge detection, symmetry axis detection, face detection and face recognition.

Olivier First State Vel and Stefan Aeberhard, [7] projected Line-Based Face Recognition underneath totally different poses In this author, propose a completely unique image-based face recognition formula that adoption a group of irregular one-dimensional line segments of second face image views because the underlying image illustration, in conjunction with a k-nearest neighbors algorithm because the line identical theme. 
A.Z. Kouzani and K. Sammut, [8] bestowed paper on invariant face recognition. A face recognition methodology that may address the variations as a result of the changes in lighting and create is bestowed during this paper. To complete illumination, associate degree formula is projected that applies associate degree embossing operator to the detected face pictures to get rid of the illumination effects.

Erik Hjelmas and B K Low, [9] an extansive and an important audit of face identification algorithms. Face detection may be a mandatory beginning in face detection systems, with the aim of contain and fatching the face region. The algorithms bestowed during this paper square measure classier as either feature-based or image-based and square measure mentioned in ways of its technical terms and performance.

Athinodoros S. Georghiades et al., [10] bestowed during this paper illumination models for face recognition containing variable lighting and create. During this analysis author appearance-based methodology for recognizing human faces in presence of variation in lighting and viewpoint.

Marian Stewart Bartlett et al., [11] bestowed Face Recognition by exploitation freelance element Analysis (ICA) generally these strategies realize a group of basis pictures and presents linear combination of facial pictures. Principal component analysis (PCA) is a example of such strategies. The premise pictures fetches by PCA on relationships between pixels and information provided by the images.

Yongsheng GAO and Maylor K.H. Leung, [12] mentioned human faces is to be recognize automatically presents a major problem to the pattern recognition analysis society. They're really inside one category of human face.

\section{Revolution of Facial Images}

\subsection{Face Detection}

An identity verification could be a system [18] that is usually used purpose by the computers for pic out somebody's from a picture that is keep within the info. The method of face recognition is take associate degree input that is given by outside and compare that image to the keep info. Info is within the style of digital image or a video frames. When we compare two images facial features is to be selected first by applying different facial feature algorithms. Face recognition system mainly used in security system biometric system used for face recognition. When we have to detect faces basically features of the face, fingerprint as well 
as jaw shape and iris recognition help to find exact image.

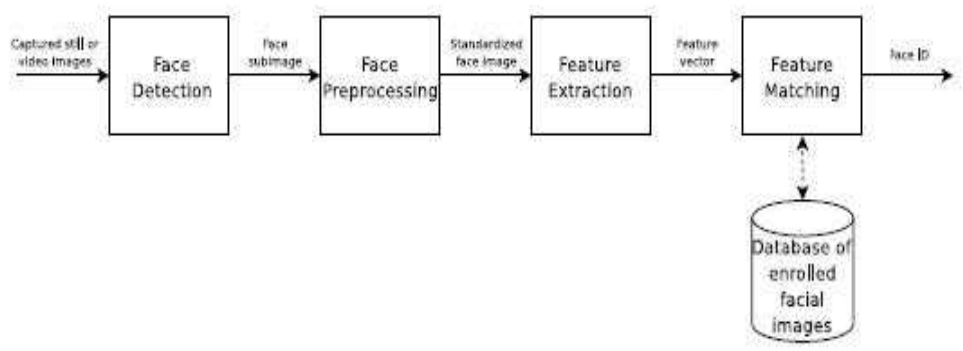

[Fig. 1] The four general steps in facial recognition

\subsection{Preprocessing}

The purpose of preprocessing step in any face detection system is to increase the FAR (False Acceptance Ratio) and to increase the speed of the system. By using preprocessing step increases computational efficiency and number of false positives.

\subsection{Feature extraction}

Feature extraction is a processing which is used after preprocessing step in feature extraction process developer/ researcher extract the feature from the human face like mouth shape, jaw line, nose, eyes, eyebrow etc. these are the features which is compared to detect exact face from the given data base.

\section{Categerorization of Face Recognition Techniques}

A category of face recognition techniques is most serious challenges faced by current face a detection technique that is facing the difficulties of variable pose handling techniques.

Image of the face varies because of rotations/moment occurs usually larger than the inter-people modification employed to identifying existence. Face recognition cross over cause, in different ends, having nice probable in several operations coping with perverse subjects, within the complete power of face recognition uses as a idle biometric technique are often enforced and appropriate. In depth achievements are place in analysis forwarded pose-invariant face recognition in past years \& lots of prominent methods are projected. 


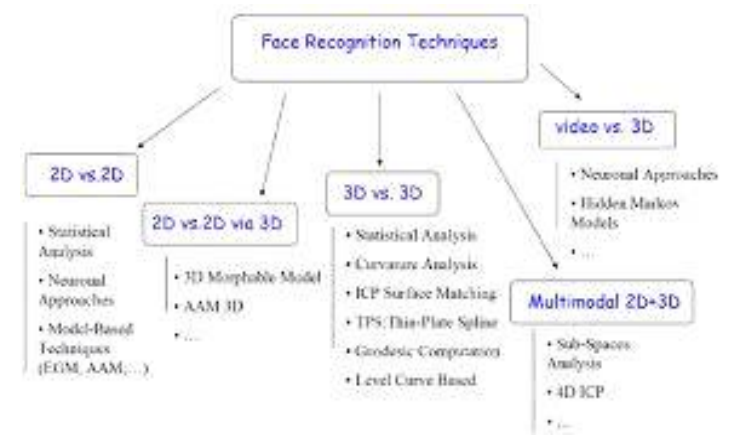

[Fig. 2] Different face recognition techniques

However, many problems for face recognition across cause static stay accessible, like shortcoming of acceptance concerning subspaces of cause variant pictures, downside intractableness in 3D face modeling, complicated in face outer part reflect called reflection mechanism, etc. In this paper a crucial discussion of researches on image-based face recognition towards cause. The present methods are systematically changed and mentioned. These methods are classified in totally different classes in line with its related methodologies by treating with its cause and its variations. Different methods, its advantages and disadvantages always considered its performances in detailed discussion. Clarifying totally different algorithms in treating handling cause variations and calculating their performances urge many positive directions for further analysis.

\subsection{General Algorithms}

A typical face recognition downside is to gradually explain someone for Associate in nursing initial (input) image with the help of it determines his/her face. The initial goal is the task will trace back recognize to over thirty years along. Then, variety of face recognition strategies are projected, among that Principal part Analysis (PCA), Fisher Discriminant Analysis (FDA called Fisher faces, Linear Discriminant Analysis (LDA) [7], Self-Organizing Map and Convolutional Network (SOM+CN), guide matching, standard PCA, Line Edge Maps (LEM), Elastic Bunch Graph Matching (EBGM), Directional Corner purpose (DCP) and native Binary Patterns (LBP) are a number of the representative works:

1) Holistic approach

2) Native approach 


\subsection{D Techniques}

According to the previous collected data researcher conclude that the majority of the result of face recognition approaches are challenging to cause variations, variety of approaches are projected to expressly treated with cause variations. 2nd methods and 3D strategies were accustomed treated or predict the looks differentiation of human faces brought by dynamical poses. In this section 2D methods are further divided into three parts as mentioned below:

1) Pose-tolerant feature extraction,

2) Real view-based matching,

3) 2nd cause transformation.

Approaches supported cause ignore feature extraction steps to realize face classifiers or pre-processing of the facial image with linear/non-linear mapping within the image which will ignore pose variations

\subsection{D Techniques}

With help of 3D models face recognition becomes one amongst palm techniques, particularly once coping accompanied cause and illumination variations. A 3D model-based approaches use to manipulate cause variations is as a result of the actual reason behind it is that human heads are $3 \mathrm{D}$ objects with clear modules and changes in sawing sides these all take places within the 3D areas. A 3D face structures play a role in face recognition may well be similar as 3D form structures, customized 3D scans, and customized 3D models reconstructed from 2nd image. Face recognition victimization customized 3D browses related to 3D face recognition and beyond the scope of this review, which is related to 2nd image-based face recognition. Here, tend to send the related interested peoples to the superb results specially related on 3D face recognition. Face recognition techniques victimization similar forms think about the similar face shape as a tool for the change over of image pixels. Customized 3D face (shape) structure are often reconstructed victimization:

1) Generic shape-based strategies

2) Feature-based 3D reconstruction

3) Image-based 3D reconstruction 
Echelon Based Pose Generalization of Facial Images Approaches

Consider facial textures (e.g., element intensities) as crucial suggestions and used it for reconstruction purpose.

\section{Proposed Work}

\subsection{Introduction to Pose Invariant}

Some of the important challenges concurrence by recent face recognition techniques facing the difficulties when treated with varied poses, i.e., face recognition in extreme rotations in depth. The facial images variations are caused due to rotations are generally bigger than the similar person variations utilized in distinctive uniqueness. Recognition of the face across results, from the opposite ends, has nice thinkable in several uses handling non-cooperative matters, during the complete face recognition power is being a yielding techniques based on biometric are often enforced and exploits. Intensive achievements are place in between the analysis in way of pose-invariant face recognition techniques in recent years and lots of different techniques are planned [2][5]. But, many problems in face recognition across cause now presents open, like lack of understanding regarding subspaces of cause different pictures, downside intractableness in face modeling with 3D, complicated face surface reflection mechanism, etc. In this paper needful survey is mentioned which is related to researches on image based face recognition across reasons. The prevailing techniques are unconditional reviewed and to be mentioned. They all are categories into completely different classes in keeping with their approaches in treating result variations. There ways, their pros/cons and performances are discuss in detail. By analyzing it completely in different ways to treating cause variations and calculated their performances, many suggested directions for further uses analysis are urged.

\subsection{Pose Invariant Techniques}

The difficult task featured by cause-unchanging algorithms for face recognition is to extract the regular temperament free from variation of poses. Basically, if additional database pictures in numerous poses are accessible, the achievements of recognizing a facial image in Associate in nursing unnoticed cause are higher. Table one represents Categorization of face recognition techniques across poses. Practically face recognition downside is to analysis establish someone in associate in nursing input image through working on his/her face. The primary commit to 
this type of task will trace back to quite thirty years past.

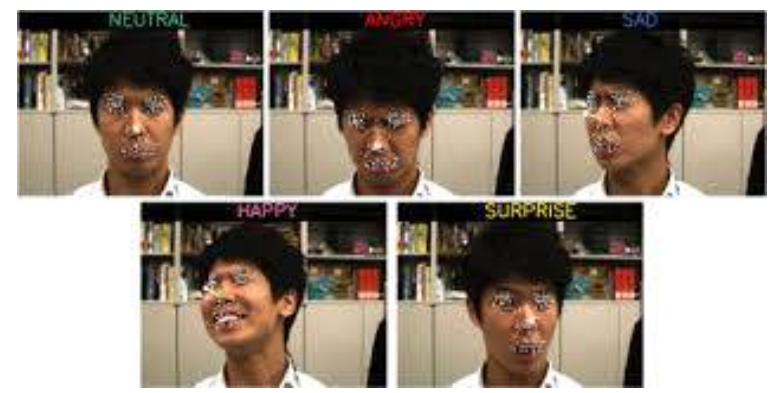

[Fig. 3] Poses of a single image

The computing world contains a heap to achieve from neural networks. Their ability to find out by example makes them terribly versatile and powerful. What is more there ought not to devise Associate in Nursing algorithmic program so as to perform a selected task; i.e. there's no ought to perceive the inner mechanisms of that task. Additionally okay fitted to real time systems as a result of their quick response and process times that are because of their parallel design. [6] Neural networks additionally contribute to alternative areas of analysis like neurology and science.

\subsection{Basic language of poses}

Face recognition across related to differentiate the face pictures in numerous poses in the computers. Researchers having an interesting field in several face recognition uses, most especially those victimization common or uncooperative fields, like police work areas. For instance, Recognition of the face is appealing in security of the field of acknowledge terrorists and save that data from boarding plane. Generally, the terrorist's faces are to be collected and hold on within the information against those travelers' faces is compared. The faces of everybody researching a safety stop are scanned. When a face match is done, cameras are switched to survey individuals with a online live video, so the jurisdictions can conforms the match and judge whether or not to prevent the individual whose face matches one within the information [1][2]. The foremost natural resolution for this task may well be to gather multiple gallery pictures all told potential causes to hide the pose variations within the captured pictures, which needs a reasonably simple face recognition algorithmic program. In several real things, however, it's boring and troublesome to gather these different gallery pictures in numerous causes and thus the power of face recognition algorithmic program to avoid pose 
variations is fascinating.

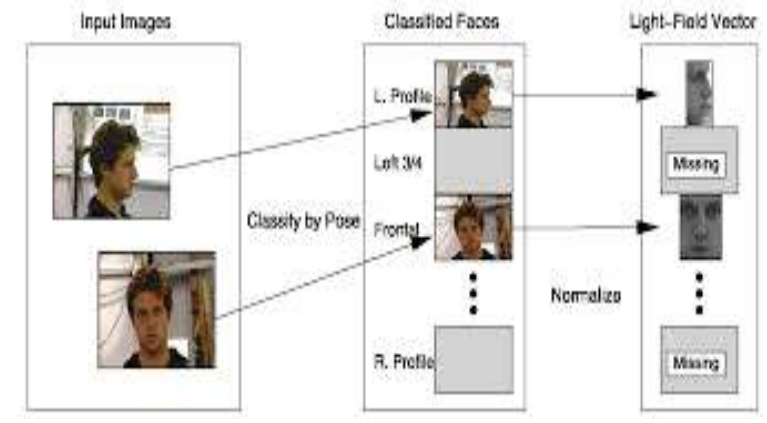

[Fig. 4] Classified images of the faces

For example, if solely a passport icon per person was hold on within the information, an honest face recognition algorithmic program ought to still be ready to perform the on top of field police work task. In this type sense, recognition of faces across cause refers to recognizing face pictures whose poses are completely away from the gallery having known pictures. If recognition of face doesn't have a honest cause avoidance, given a front passport icon, the system seems to want cooperative subjects which look directly at the face.

\section{Applications of Pose Invariant Technology}

1. Face Identification: To establish the face with the assistance of input image compare it with the hold on info.

2. Surveillance: Surveillance primarily used for the aim of perceptive a district. They typically connected to a recording device or IP network, and should be watched by a watchman or enforcement officer. Police work is that the watching of the behavior, activities, or different dynamic info, typically of individuals for the aim of influencing, managing, directing, or protective them.

3. Access Control: Permission to access a resource is named authorization. And therefore the management over to access something referred to as access management

4. Non-public Security: Private security guards make sure the safety of individuals, property and places. Here with the assistance of face recognition system we have a tendency to powerfully secure our information in private.

5. General identity verification: Identity verification is the main step in all the field with the help of all biometric check as well as document verification. In this phase face recognition play a very important role. 
6. Image database investigation: Image database, which is basically used by the police, forensic or in CID department, used for the investigation purpose. In that case face identification is very important.

7. Easy people tagging: Tagging basically used in social networking site with the help of face recognition.

8. Gaming: In gaming all biometric functions are used face recognition is one of them.

\section{Challenges Facing in Face Recognition}

Below mentioned challenges in field of face recognition system developer and researchers basically work on the following elaborated points.

(1) Facial aging: In the human body, many changes occurs with respect to the time and it can also be noticed on the face of the person due to hormonal and biological changes. This is a challenge for researchers to make our system sufficiently smart to overcome this problem. As a result we don't have to update our system with time and our database can never be outdated.

(2) Accessory used during input images: In some cases, peoples uses many accessories on their faces like goggles, specs, some type of nails in nose, boys can have beard etc. these factors can affect the desired result which is an important challenge for this technology.

(3) Pose invariant: Sometimes the person standing in front of the camera makes some angle which can be horizontal or vertical which effects the desired result. In real means we are developing a technology which should be user friendly. Being a user friendly technology, it should have a wide range of detection but at present it can be allow the pose variation up to a very limited range.

(4) Lighting conditions: The lightening conditions like background light, brightness, contrast, shadow etc. are not always same for the input. So the system must be smart enough to adjust these changes accordingly.

(5) Plastic surgery: In some special cases, due to some accidental reasons or so, many peoples have gone through the plastic or cosmetic surgery as a result of which the inputs of their faces can be unknown for the system. But for researchers it is a challenge to make the system able to detect the same person after the above mentioned changes.

(6) Accidental face detection: Due to some emergency conditions or some accidents, the face of the victim damaged enough to lose his identity. If the researchers can do some needful and 
if they could make the system able to detect these faces, It will be the milestone for the forensic field.

\section{Further Work}

After the deep study of literature survey and the challenges on face recognition technology, it is to be noted that there are many challenges in this field and if the researchers could find the solutions of these challenges in future, it can surely effect the accuracy and precision of the system positively.

\section{Conclusion}

Pose could be a difficult and unresolved drawback in face recognition. and also the create drawback is sometimes plus alternative factors to conjointly have an effect on the performance of sensible face recognition systems.3D create standardization strategies estimate solely the create and form of a theme from one or multiple 2nd face pictures, whereas the feel data is directly mapped from the input 2nd image to the 3D model. The pictures from the rough 3D model are consequently realistic in look. Coordinates of dense facial landmarks, to estimate create and form parameters. The error in create and form estimation leads to undesirable artifacts within the succeeding texture mapping and face synthesis operations, that adversely AN acts face recognition [2][6][9]. The pose-invariant face recognition strategies exploitation 2nd techniques are classified into 3 teams, i.e., real view-based matching, create transformation in image area, and create transformation in feature area.

\section{References}

[1] R. Brunelli and T. Poggio, Face recognition: features versus templates, IEEE Transactions on PAMI, (1993), Vol.15, No.10, pp.1042-1052.

[2] J. K. Wu and A. D. Narasimhalu, Identifying faces using multiple retrievals, IEEE MultiMedia, (1994), Vol.1, No.2, pp.27-38.

[3] N. F. Troje and H. H. Bülthoff, Face recognition under varying poses: The role of texture and shape, Vision Research, (1996), Vol.6, No.1, pp.1761-1771.

[4] S. H. Lin, S. Y. Kung and L. J. Lin, Face Recognition/Detection By Probabilistic Decision-Based Neural 
Network, IEEE, (1997), Vol.8, No.1, pp.114-132.

[5] J. Ben-Arie and D. Nandy, A volumetric/iconic frequency domain representation for objects with application for pose invariant face recognition, IEEE TPAMI, (1998), Vol.20, No.5, pp.449-457.

[6] T. Kondo and H. Yan, Automatic Human Face Detection and Recognition under Non-Uniform Illumination, Pattern Recognition, (1999), Vol.32, No.10, pp.1707-1718.

[7] O. D. Vel and S. Aeberhard, Line-Based Face Recognition Under Varying Pose, IEEE TPAMI, (1999), Vol.1, No.3, pp.1081-1088.

[8] A. Z. Kouzani, F. He and K. Sammut, Towards Invariant Face Recognition, Information Sciences, (2000), Vol.123, No.1-2, pp.75-101.

[9] E. Hjelmås and B. K. Low, Face Detection: A Survey, Computer Vision and Image Understanding, (2001), Vol.83, No.3, pp.236-274.

[10] R. Tyagi, G. S Tomar and L. Shrivastava, Unconstrained Face Recognition Quality: A Review, International Journal of Signal Processing, Image Processing and Pattern Recognition, (2016), Vol.9, No.11, pp.199-210.

[11] M. S. Bartlett, J. R. Movellan and T. J. Sejnowski, Face Recognition by Independent Component Analysis, IEEE Trans Neural Netw., (2002), Vol.13, No.6, pp.1450-1464.

[12] Y. Gao and M. K. H. Leung, Face Recognition Using Line Edge Map, IEEE TPAMI, (2002), Vol.4, No.6, pp.764-779.

[13] C. Liu and H. Wechsler, Independent Component Analysis Of Gabor Features For Face Recognition, IEEE Transactions on Neural Networks, (2003), Vol.14, No.4, pp.919-928.

[14] V. Blanz and T. Vetter, Face recognition based on fitting a 3D morphable model, IEEE TPAMI, (2003), Vol.25, No.9, pp.1063-1074.

[15] X. He, S. Yan, Y. Hu, P. Niyogi and H. Zhang, Face Recognition Using Laplacian faces, IEEE TPAMI, (2005), Vol.27, No.3, pp.328-340.

[16] D. Dubey, G. S Tomar and H. Kim, Scrutinized Study on Face Recognition by Pose invariant Methodology, International Journal of future Generation Communication and Networking, (2016), Vol.9, No.9, pp.333-342.

[17] D. Dubey and G. S Tomar, Deep Perusal of Human Face Recognition Algorithms from Facial Snapshots, International Journal of Signal Processing, Image Processing and Pattern Recognition, Image Processing and Pattern Recognition, (2016), Vol.9, No.9, pp.103-112. 